\title{
The effect of polymyxin B hemoperfusion on modulation of human leukocyte antigen DR in severe sepsis patients
}

\author{
Nattachai Srisawat ${ }^{1,2,3^{*}}$, Somkanya Tungsanga ${ }^{1}$, Nuttha Lumlertgul ${ }^{1,2}$, Chalermchai Komaenthammasophon ${ }^{1,2}$, \\ Sadudee Peerapornratana 1,2,3, Nicha Thamrongsat ",2, Khajohn Tiranathanagul', Kearkiat Praditpornsilpa', \\ Somchai Eiam-Ong ${ }^{1}$, Kriang Tungsanga ${ }^{1}$ and John A. Kellum ${ }^{3}$
}

\begin{abstract}
Background: Recent randomized trials have not found that polymyxin B hemoperfusion (PMX-HP) improves outcomes for patients with sepsis. However, it remains unclear whether the therapy could provide benefit for highly selected patients. Monocyte human leukocyte antigen (mHLA-DR) expression, a critical step in the immune response, is decreased during sepsis and leads to worsening sepsis outcomes. One recent study found that PMX-HP increased mHLA-DR expression while another found that the treatment removed HLA-DR-positive cells.

Methods: We conducted a randomized controlled trial in patients with blood endotoxin activity assay (EAA) level $\geq$ 0.6. Patients in the PMX-HP group received a 2-h PMX-HP treatment plus standard treatment for 2 consecutive days. Patients in the non-PMX-HP group received only standard treatment. The primary outcome compared the groups on median change in mHLA-DR expression between day 3 and baseline. Secondary outcomes compared the groups on the mean or median change in CD11b expression, neutrophil chemotaxis, presepsin, cardiovascular Sequential Organ Failure Assessment (CVS SOFA) score, vasopressor dose, and EAA level between day 3 and baseline. We further compared the groups on mortality, ICU-free days, ventilator-free days, dialysis dependence status, renal recovery, serum creatinine, vasopressor-free days, and major adverse kidney events (MAKE 28), measured on day 28.
\end{abstract}

Results: Fifty-nine patients were randomized to PMX-HP $(n=29)$ and non-PMX-HP $(n=30)$ groups. At baseline, mHLADR expression, CD11b, neutrophil chemotaxis, and clinical parameters were comparable between groups. The median change in mHLA-DR expression between day 3 and baseline was higher in PMX-HP patients than in patients receiving standard therapy alone $(P=0.027)$. The mean change in CD11 b between day 3 and baseline was significantly lower in the PMX-HP group than in the non-PMX-HP group $(P=0.002)$. There were no significant changes from baseline in neutrophil chemotaxis, presepsin, CVS SOFA scores, vasopressor doses, or EAA level between groups. On day 28 after enrollment, mortality, ICU-free days, ventilator-free days, dialysis dependence status, renal recovery, serum creatinine, vasopressor-free days, and MAKE 28 were comparable between groups.

Conclusion: PMX-HP improved mHLA-DR expression in severe sepsis patients. Future studies should examine the potential benefit of PMX-HP in patients with low mHLA-DR expression.

Trial registration: ClinicalTrials.gov, NCT02413541. Registered on 3 March 2015.

Keywords: Immunoparalysis, Severe sepsis/septic shock, Polymyxin B hemoperfusion

\footnotetext{
* Correspondence: drnattachai@yahoo.com

'Division of Nephrology, Department of Medicine, Faculty of Medicine,

Chulalongkorn University, Bangkok 10330, Thailand

${ }^{2}$ Excellence Center for Critical Care Nephrology, King Chulalongkorn

Memorial Hospital, Thai Red Cross Society, Bangkok, Thailand

Full list of author information is available at the end of the article
}

(c) The Author(s). 2018 Open Access This article is distributed under the terms of the Creative Commons Attribution 4.0 International License (http://creativecommons.org/licenses/by/4.0/), which permits unrestricted use, distribution, and reproduction in any medium, provided you give appropriate credit to the original author(s) and the source, provide a link to the Creative Commons license, and indicate if changes were made. The Creative Commons Public Domain Dedication waiver (http://creativecommons.org/publicdomain/zero/1.0/) applies to the data made available in this article, unless otherwise stated. 


\section{Background}

Although there has been substantial improvement of care through the use of potent antibiotics and sepsis care bundles, severe sepsis/septic shock is still the leading cause of death worldwide [1-3].

For more than 25 years, polymyxin B hemoperfusion (PMX-HP) has been introduced as one of the treatments for sepsis in Japan, and recently in Europe and Asia [4, 5]. It was reported that polymyxin B could neutralize the various biologic activities of endotoxins [6]. Data from a noncontrolled clinical study demonstrated that PMX-HP might improve sepsis outcomes by diminishing the negative effects of hypotension [7], reducing serum cytokine levels [8] or improving monocyte mRNA expression $[9,10]$. However, the molecular mechanism of PMX-HP and its usefulness is not yet clear and has never been tested in a randomized controlled trial.

During the early phase of sepsis there is a massive release of proinflammatory cytokines which are responsible for organ dysfunction and tissue hypoperfusion. Simultaneously there is an anti-inflammatory response to balance the body's homeostasis and to avoid the excessive effect of a proinflammatory response. However, this effect might lead to a state of immunoparalysis and cause secondary infections from opportunistic pathogens [11]. Unfortunately, we still lack an effective treatment to improve the immunoparalysis state.

The monocyte/macrophage system plays one of the major roles in host defense. The main functions of monocytes, antigen expression, and cytokine production are mediated by certain surface molecules from major histocompatibility complex (MHC) class II, such as monocyte human leukocyte antigen (mHLA-DR). mHLA-DR allows antigen presentation to $\mathrm{T}$ cells and is crucial for the initiation of the cascade of the immune response during sepsis [12]. Therefore, the diminished expression of mHLA-DR could represent a state of immunoparalysis and would lead to higher risk of secondary infection. A recent report also showed that low mHLA-DR expression might relate to higher mortality rates [13]. However, recent concepts have been put forth including "tolerance" which posit that suppression of the immune system can be beneficial for limiting tissue damage. Thus, it remains unclear whether to use drugs to boost the immune system [14].

We conducted a randomized controlled trial to evaluate the effect of PMX-HP on mHLA-DR expression. We also explored the effect of PMX-HP on CD11b expression on the neutrophil, neutrophil chemotaxis, cardiovascular Sequential Organ Failure Assessment (CVS SOFA) score, vasopressor dose, and endotoxin level. Furthermore, mortality, intensive care unit (ICU)-free days, ventilator-free days, dialysis dependence status, renal recovery, and serum creatinine were compared on day 28 .

\section{Methods}

The study was conducted at King Chulalongkorn Memorial Hospital, Bangkok, Thailand in five ICUs: two medical, one coronary care, and two surgical. The study was conducted between February 2014 and July 2017. The protocol was approved by the Faculty Ethical Committee (IRB No. 578/56) and registered at ClinicalTrials.gov (NCT02413541). Informed consent was obtained from each patient or the patient's surrogate.

\section{Study population}

Patients were eligible to participate in the trial if they were 18 years of age or older, and were diagnosed with severe sepsis or septic shock according to the consensus definition of the American College of Chest Physicians (ACCP)/Society of Critical Care Medicine (SCCM) Consensus Conference Committee [15] regardless of renal function or blood pressure. Patients were not eligible if they had one of the following: expected to die within $24 \mathrm{~h}$, pregnant, white blood cell count less than $500 / \mathrm{mm}^{3}$, platelet count less than $30 \times 10^{3} / \mathrm{mm}^{3}$, uncontrolled coagulopathy, terminal illness with Do-Not-Resuscitate will, allergic reaction to PMX-HP, or organ transplantation.

\section{Randomization}

On admission to the ICU venous blood was collected for endotoxin activity assay (EAA). Patients with EAA levels of 0.6 or greater were randomly assigned by blocked randomization into two groups: the PMX-HP group and the non-PMX-HP group. Although the allocation sequence was concealed it was not possible to conceal the intervention among the two groups. However, during the analysis the investigators were blinded to the group allocation of each subject.

\section{Study treatments}

All patients were treated with the standard treatment according to the Surviving Sepsis Campaign guideline [16] by physicians with experience in extracorporeal blood purification techniques. Venous access was undergone using a double-lumen catheter. Patients in the PMX-HP group received a 2 -h PMX-HP treatment plus standard treatment for 2 consecutive days after enrollment. The hemoperfusion was done with adsorbent columns consisting of $5 \mathrm{mg}$ of polymyxin B per gram of polystyrene fiber (Toraymyxin; Toray Industries, Tokyo, Japan). Access to blood for PMX-HP treatment was obtained through a double-lumen catheter inserted into the central venous by Seldinger's method. The blood flow rate was set at $120 \mathrm{ml} / \mathrm{min}$. Heparin infusion was the 
standard anticoagulant. If a patient had contraindication to heparin we used the nonanticoagulant strategy. Neither volume replacement nor ultrafiltration was conducted during the hemoperfusion process.

\section{Endotoxin activity assay}

We used the chemiluminescent-based endotoxin activity assay (Spectral Diagnostics, Ontario, Canada) as described elsewhere [17]. This assay is based on the detection of enhanced respiratory burst activity in neutrophils following their priming by complexes of endotoxin and a specific anti-endotoxin antibody. Briefly, $40 \mu \mathrm{l}$ of whole blood were incubated with zymosan and anti-endotoxin antibody. The immune complex between endotoxin and specific antibody potentiates the zymosan-driven reactive oxygen species (ROS) production by neutrophils. The amount of ROS released indicates the relative amount of endotoxin in blood [17-19]. ROS were determined by chemiluminescent assay normalized for the neutrophils' ability to produce ROS.

\section{Measurement of $\mathrm{mHLA}-\mathrm{DR}$ expression, CD11b expression on neutrophils, and neutrophil chemotaxis Neutrophil isolation}

Whole blood was collected by venipuncture into heparinized tubes. Polymorphonuclear cells (PMN) and mononuclear cells were extracted by one-step centrifugation with Polymorphprep ${ }^{\mathrm{m}}$, which contained sodium diatrizoate and an $8 \%(\mathrm{w} / \mathrm{v})$ polysaccharide solution (AxisShield, Norway). Fresh blood was layered onto PolymorphPrep ${ }^{\text {tw }}$ in a 1:1 ratio and centrifuged for $30 \mathrm{~min}$ at $500 \times g(1700-1800 \mathrm{rpm})$. This density gradient allows separation of both monocytes and PMN. Following separation, the monocyte and PMN layer was removed with a sterile pipette and washed with RPMI 1640 by centrifugation at $800 \times g(2000 \mathrm{rpm})$ for $5 \mathrm{~min}$ to remove residual PolymorphPrep ${ }^{\mathrm{Tx}}$. Contaminating erythrocytes were remove by resuspending the cell pellet in ammonium chloride lysis buffer in 9:1 ratio with RPMI 1640 for 3 min. Cells were pelleted at $800 \times g$ for 5 min and resuspended in RPMI 1640 supplemented with 5-10\% (v/v) human AB serum (or fetal bovine serum).

\section{Flow cytometry for detecting $m$ HLA-DR expression and CD11b expression on neutrophils}

The CD11b expression on neutrophils and HLA-DR expression on monocytes were measured using monoclonal antibody staining and flow cytometry. Neutrophils and monocytes were collected and then incubated with allophycocyanin (APC)-conjugated CD11b (IgG1; Beckman Coulter, France) and with phycoerythrin (PE)-conjugated HLA-DR (IgG1; Beckman Coulter) for $30 \mathrm{~min}$, respectively. After incubation the samples were washed twice in PBS without calcium or magnesium and fixed with $4 \%$ paraformaldehyde. All samples were run within 7 days of collection by a flow cytometer (BD LSR II; BD Biosciences, USA) using acquisition and analysis software (BD FACSDiva; BD Biosciences).

\section{Neutrophil chemotaxis}

The chemotaxis assay was performed using Hanging Millicell inserts (Merck Millipore, Germany). Twenty-four-well tissue culture well plates were coated with poly(2-hydroxyethyl methacrylate) to prevent cell adhesion following transmigration. Then $800 \mu \mathrm{l}$ RPMI 1640 with chemoattractant $\left(10^{-9} \mathrm{~mol} / \mathrm{L}\right.$ formyl methionyl-phenylalanine (FMLP)) was added to each well. The hanging inserts with a $3-\mu \mathrm{m}$ pore-size filter at their base and $1 \times 10^{6}$ neutrophils in $200 \mu \mathrm{l}$ culture media were added into the hanging inserts and incubated at $37{ }^{\circ} \mathrm{C}$ in a $5 \% \mathrm{CO}_{2}$ incubator for $90 \mathrm{~min}$. The hanging inserts were then removed and migrated neutrophils in each well were counted. The count number of migrated neutrophils was calculated as a percentage of the total number of cells originally added [20].

\section{Measurement of presepsin}

Presepsin concentrations in plasma were measured using a compact fully automated immunoassay analyzer (PATHFASTTM System; LSI Medience Corporation, Japan/Mitsubishi Chemical Europe), based on a noncompetitive chemiluminescence enzyme immunoassay [21].

\section{Data collection}

Clinical data and laboratory testing were collected on days $1,2,3,7,14,21$, and 28 after enrollment. mHLA-DR expression, CD11b expression, neutrophil chemotaxis, and EAA levels were assessed on days 1 and 3. Clinical severity was assessed on the aforementioned days using the SOFA score [22]. The amount of vasoactive agents used was measured by the inotropic score $[23,24]$ and the vasopressor index $[25,26]$.

\section{Outcomes}

The primary outcome was the change in mHLA-DR expression, measured as mHLA-DR on day 3 after enrollment minus mHLA-DR at baseline. Secondary outcomes, defined as change between day 3 and baseline, included: CD11b expression on neutrophil, neutrophil chemotaxis, CVS SOFA score, vasopressor dose, and EAA level. Finally, clinical outcomes measured on day 28 included: mortality, ICU-free days, ventilator-free days, dialysis dependence status, renal recovery, serum creatinine, vasopressor-free day, and major adverse kidney events (MAKE) by day 28. Primary and secondary outcomes were measured on those alive on day 3 . Outcomes on day 28 were measured in those still alive at the start of the day. 


\section{Statistical analysis}

According to Ono et al. [10], in order to detect a $15 \%$ difference in mHLA-DR expression between groups a sample size of 60 was needed: 30 patients for the PMX-HP group and 30 patients for the non-PMX-HP group.

Statistical analyses were performed using Stata version 15.0, with statistical significance set at $P<0.05$. Comparisons between groups were performed with Fisher's exact test for categorical variables and using Student's $t$ test or Kruskal-Wallis one-way analysis of variance by ranks for continuous variables. The main and secondary outcomes, calculated as change from baseline, were tested between groups with either Student's $t$ test or Kruskal-Wallis test depending on their distribution. Kaplan-Meier survival curves were used to estimate the cumulative mortality rates in the two groups. Categorical data are summarized as counts and percentage. Continuous data are summarized as mean (standard deviation) or median (25th percentile, 75 th percentile).

\section{Results}

\section{Cohort characteristics}

We recruited 141 severe sepsis/septic shock patients. Sixty had EAA $\geq 0.6$ and were randomized equally to the PMX-HP and non-PMX-HP groups (Fig. 1). One patient from the PMX-HP group had incomplete data and was left out of the analysis. All 29 patients in the PMX-HP group received two sessions of PMX-HP. Two patients had circuit interruption, one due to technical problems and another due to circuit clotting. Baseline characteristics between the two groups were similar except for the rate of renal replacement therapy (RRT), which was higher in the PMX-HP group (93\% vs $69 \%, P=0.019$ ) (Table 1 ). CRRT was the most common type of RRT in both groups (77.8\% for PMX-HP group, and 70\% for non-PMX-HP group), followed by sustained low-efficiency dialysis. CVVHDF was the only mode of CRRT used in the study.

Isolated microorganisms and site of infection are presented in Table 2. Gram-negative bacteria were the predominant organisms while the respiratory tract was the most common site of infection.

\section{Primary outcome}

Baseline median mHLA-DR expression did not differ between the PMX-HP and non-PMX-HP groups. In the PMX-HP group the median mHLA-DR expression significantly increased from $33.4 \%$ (25th and 75th percentiles $27.4 \%, 40.7 \%)$ on day 1 to $39.2 \%(27.1 \%, 40.1 \%)$ on day $3(P=0.025)$ (Table 3 , Fig. 2$)$. In the non-PMX-HP group there was no change in the median mHLA-DR expression between day 1 and day 3: $30.0 \%$ (15.8\%, $36.6 \%)$ vs $30.7 \%(19.5 \%, 36.8 \%)(P=0.13)$. The median mHLA-DR expression on day 3 was significantly higher in the PMX-HP group than in the non PMX-HP group $(P=0.027)$. The median change in mHLA-DR expression between day 3 and day 1 was significantly higher in the PMX-HP group than in the non PMX-HP group $(P=$ 0.027). At each time point, there was no significant difference of monocyte count and neutrophil count between the two groups. (Additional file 1: Table S1).

\section{Secondary outcomes}

In the PMX-HP group, the mean CD11b expression on neutrophils did not significantly change between days 1 and 3 (from $15.4 \%$ to $13.7 \%, P=0.30$ ), while in the non-PMX-HP group it significantly increased from day 1 to day 3 (from $14.3 \%$ to $16.2 \%, P=0.001$ ). The mean change in $\mathrm{CD} 11 \mathrm{~b}$ between day 3 and day 1 was

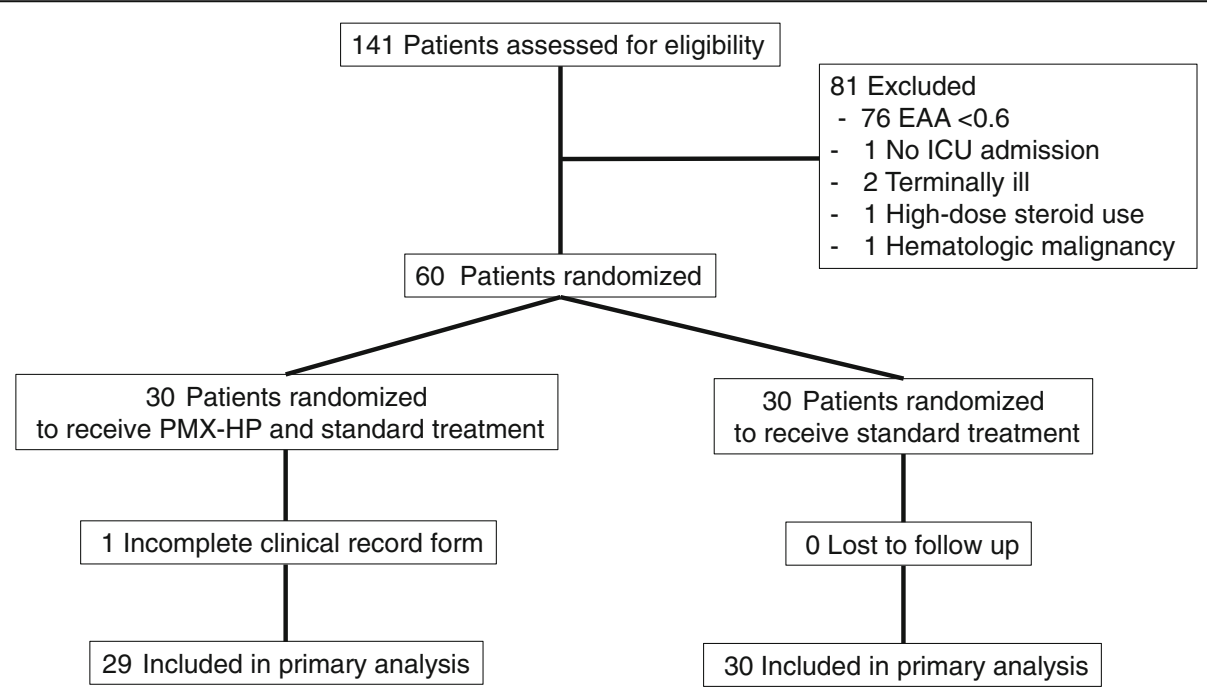

Fig. 1 Flow diagram of patient allocation. EAA endotoxin activity assay, ICU intensive care unit, PMX-HP polymyxin B hemoperfusion 
Table 1 Baseline characteristics of treatment groups

\begin{tabular}{|c|c|c|c|}
\hline Characteristic & $\begin{array}{l}\text { PMX-HP group } \\
(n=29)\end{array}$ & $\begin{array}{l}\text { Non-PMX-HP } \\
\text { group }(n=30)\end{array}$ & $P$ value \\
\hline Age (years), mean (SD) & $70.2(13.0)$ & $67.4(14.6)$ & 0.43 \\
\hline Male, $n(\%)$ & $23(79)$ & $17(57)$ & 0.06 \\
\hline $\mathrm{BMI}\left(\mathrm{kg} / \mathrm{m}^{2}\right)$, mean $(\mathrm{SD})$ & $24.2(4.3)$ & $23.5(4.7)$ & 0.62 \\
\hline $\begin{array}{l}\text { Baseline serum creatinine } \\
(\mathrm{mg} / \mathrm{dl}) \text {, median }(\mathrm{Q} 1, \mathrm{Q} 3)\end{array}$ & $1.0(1.0,1.1)$ & $1.0(0.8,1.1)$ & 0.48 \\
\hline $\begin{array}{l}\text { BUN at enrollment }(\mathrm{mg} / \mathrm{dl}) \text {, } \\
\text { median }(\mathrm{Q} 1, \mathrm{Q} 3)\end{array}$ & $41(26,63)$ & $41(22,62)$ & 0.65 \\
\hline $\begin{array}{l}\text { Serum creatinine at } \\
\text { enrollment }(\mathrm{mg} / \mathrm{dll}) \text {, } \\
\text { median }(\mathrm{Q} 1, \mathrm{Q} 3)\end{array}$ & $2.2(1.6,3.1)$ & $2.6(1.8,4.0)$ & 0.34 \\
\hline $\mathrm{AKI}, n(\%)$ & & & 0.16 \\
\hline Non-AKI & $4(14)$ & $5(17)$ & \\
\hline Stage 1 & $8(28)$ & $4(13)$ & \\
\hline Stage 2 & $9(31)$ & $5(17)$ & \\
\hline Stage 3 & $8(28)$ & $16(53)$ & \\
\hline RRT, $n(\%)$ & $27(93)$ & $20(69)$ & 0.02 \\
\hline CRRT & $21(72 \%)$ & $14(48 \%)$ & \\
\hline SLED & $6(21 \%)$ & $4(14 \%)$ & \\
\hline Peritoneal dialysis & $0(0 \%)$ & $2(7 \%)$ & \\
\hline SOFA score, mean (SD) & $13.8(4.4)$ & $13.3(5.0)$ & 0.71 \\
\hline \multicolumn{4}{|l|}{ Type of fluid, $n(\%)$} \\
\hline Crystalloid & $23(79)$ & $23(77)$ & 0.81 \\
\hline Colloid & $15(52)$ & $13(43)$ & 0.52 \\
\hline $\begin{array}{l}\text { Inotrope/vasopressor } \\
\text { use, } n(\%)\end{array}$ & $25(86)$ & $25(83)$ & $>0.99$ \\
\hline Adrenaline & $8(28)$ & $6(20)$ & 0.49 \\
\hline Noreadrenaline & $20(69)$ & $22(73)$ & 0.71 \\
\hline Dopamine & $8(28)$ & $4(13)$ & 0.17 \\
\hline Dobutamine & $4(14)$ & $3(10)$ & 0.71 \\
\hline Vasopressin & $0(0)$ & $0(0)$ & $>0.99$ \\
\hline Terlipressin & 1 (3) & $0(0)$ & 0.49 \\
\hline $\begin{array}{l}\text { Inotropic score } \\
(\mu \mathrm{g} / \mathrm{kg} / \mathrm{min}), \\
\text { median (Q1, Q3) }\end{array}$ & $36(7,70)$ & $30(2,83)$ & 0.96 \\
\hline $\begin{array}{l}\text { Vasopressor index } \\
(\mu \mathrm{g} / \mathrm{kg} / \mathrm{min} / \mathrm{mmH} \mathrm{Hg}) \\
\text { median }(\mathrm{Q} 1, \mathrm{Q} 3)\end{array}$ & $0.4(0.1,1.1)$ & $0.4(0.1,1.1)$ & 0.93 \\
\hline $\begin{array}{l}\text { EAA at enrollment, } \\
\text { median }(\mathrm{Q} 1, \mathrm{Q} 3)\end{array}$ & $0.8(0.7,1.0)$ & $0.7(0.7,0.9)$ & 0.24 \\
\hline $\begin{array}{l}\text { Presepsin }(\mathrm{pg} / \mathrm{ml}) \\
\text { median }(\mathrm{Q} 1, \mathrm{Q} 3)\end{array}$ & $\begin{array}{l}5514(4088 \\
10,513)\end{array}$ & $\begin{array}{l}5030(2459 \\
10,734)\end{array}$ & 0.47 \\
\hline
\end{tabular}

Data represent sample size and column percentage unless otherwise noted $P M X-H P$ polymyxin B hemoperfusion, $S D$ standard deviation, $B M I$ body mass index, Q1 25th percentile, Q3 75th percentile, BUN blood urea nitrogen, AKI acute kidney injury, RRT renal replacement therapy, CRRT continuous renal replacement therapy, SLED sustained low-efficiency dialysis, CVS SOFA cardiovascular Sequential Organ Failure Assessment, EAA endotoxin activity assay

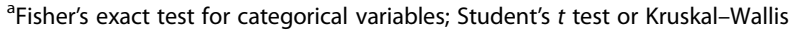
test for continuous variables, Italic data represent $P$ value statistically significant
Table 2 Isolated microorganisms by treatment group

\begin{tabular}{|c|c|c|c|c|}
\hline Characteristic & $\begin{array}{l}\text { PMX-HP group } \\
(n=29)\end{array}$ & $\begin{array}{l}\text { Non PMX-HP } \\
\text { group }(n=30)\end{array}$ & $\begin{array}{l}\text { Overall } \\
(N=59)\end{array}$ & $P$ value \\
\hline \multicolumn{5}{|l|}{ Organism } \\
\hline $\begin{array}{l}\text { Gram-positive } \\
\text { bacteria }\end{array}$ & $7(23 \%)$ & $2(7 \%)$ & $9(15 \%)$ & 0.15 \\
\hline $\begin{array}{l}\text { Gram-negative } \\
\text { bacteria }\end{array}$ & $13(43 \%)$ & $18(60 \%)$ & 31 (53\%) & 0.20 \\
\hline Fungal infection & $1(3 \%)$ & $2(7 \%)$ & $3(5 \%)$ & 1.00 \\
\hline Mycobacterium & $1(3 \%)$ & $0(0 \%)$ & $1(2 \%)$ & 1.00 \\
\hline Other & $0(0 \%)$ & $1(3 \%)$ & $1(2 \%)$ & 1.00 \\
\hline \multicolumn{5}{|l|}{ Site of infection } \\
\hline $\begin{array}{l}\text { Central nervous } \\
\text { system }\end{array}$ & 2 & 0 & $2(3 \%)$ & 0.14 \\
\hline Respiratory tract & 6 & 12 & $18(31 \%)$ & 0.11 \\
\hline $\begin{array}{l}\text { Gastrointestinal } \\
\text { tract }\end{array}$ & 9 & 6 & $15(25 \%)$ & 0.33 \\
\hline $\begin{array}{l}\text { Genitourinary } \\
\text { tract }\end{array}$ & 3 & 3 & $6(10 \%)$ & 0.97 \\
\hline Musculoskeletal & 0 & 3 & $3(5 \%)$ & 0.08 \\
\hline $\begin{array}{l}\text { Systemic } \\
\text { infection }\end{array}$ & 5 & 4 & $9(15 \%)$ & 0.68 \\
\hline Other & 5 & 3 & $8(14 \%)$ & 0.42 \\
\hline
\end{tabular}

Data represent sample size and column percentage

$P M X-H P$ polymyxin $B$ hemoperfusion

${ }^{a}$ Fisher's exact test for categorical variables; Student's $t$ test or Kruskal-Wallis test for continuous variables

significantly lower in the PMX-HP group than in the non-PMX-HP group $(P=0.002)$ (Table 3, Fig. 2$)$.

In the PMX-HP group, the mean neutrophil chemotaxis significantly increased from day 1 to day 3 (from $42.8 \%$ to $47.9 \%, P=0.03$ ), while in the non-PMX-HP group there was no change (from $42.3 \%$ to $46.2 \%, P=0.73$ ). However, we did not detect a difference between groups in the mean change from baseline $(P=0.07)$. There was improvement in the CVS SOFA score in the PMX-HP group $(P=0.003)$ but not in the non-PMX-HP group $(P=0.26)$. However, we did not detect a difference between groups in the median change from baseline $(P=0.16)$.

We did not notice a difference between groups in the median change from baseline in presepsin, inotropic score, vasopressor dose, or noradrenaline dose. There was no significant change in EAA levels from baseline within or between groups. We also did not see a difference between groups in mortality (days 7 or 28), ICU-free days, ventilator-free days, dialysis dependence status, renal recovery, serum creatinine on day 28 , vasopressor-free days, and MAKE 28 (Table 4).

\section{Adverse events}

During the study there were no adverse events due to bleeding or hypo/hypertension, and no events indicative 


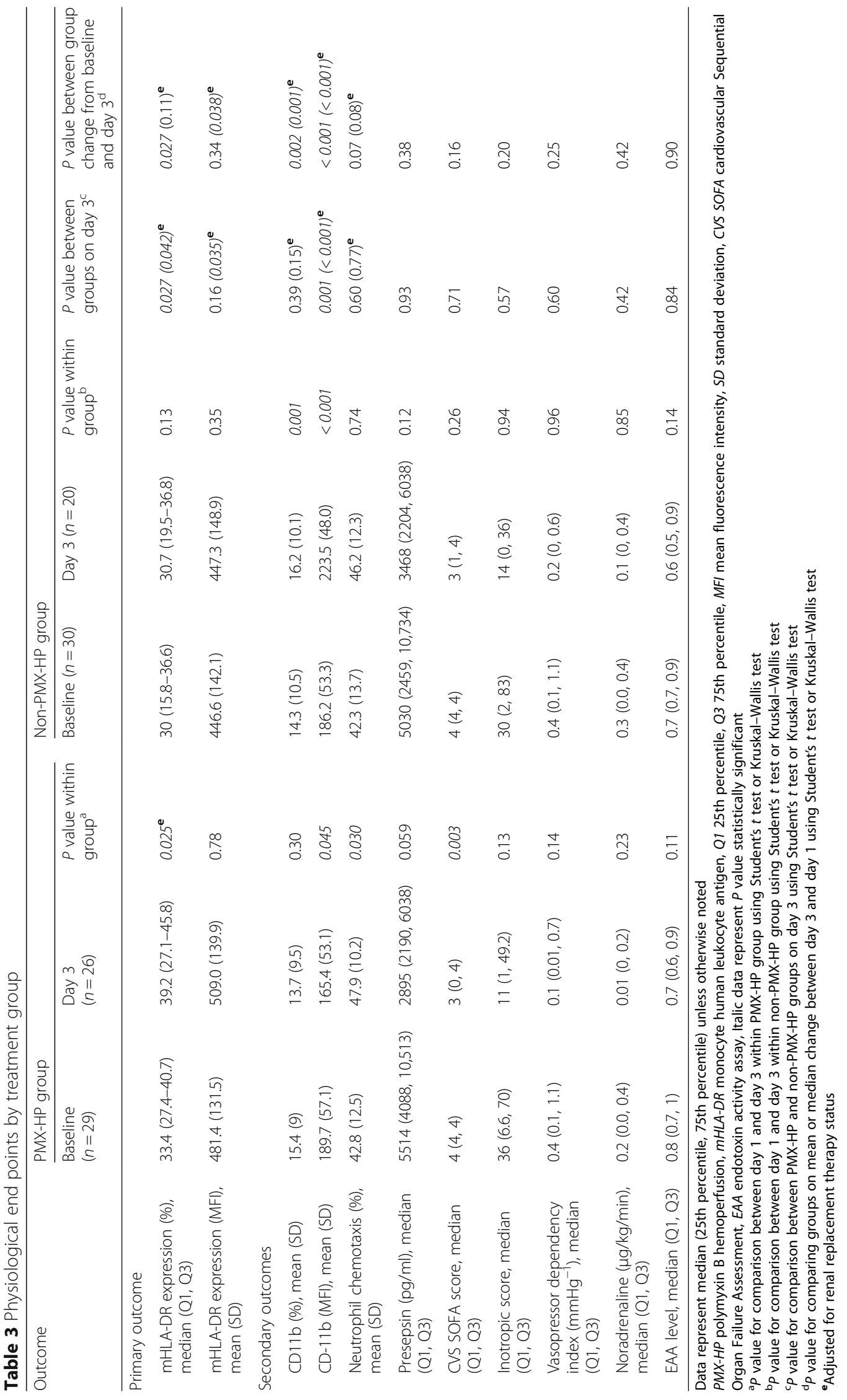




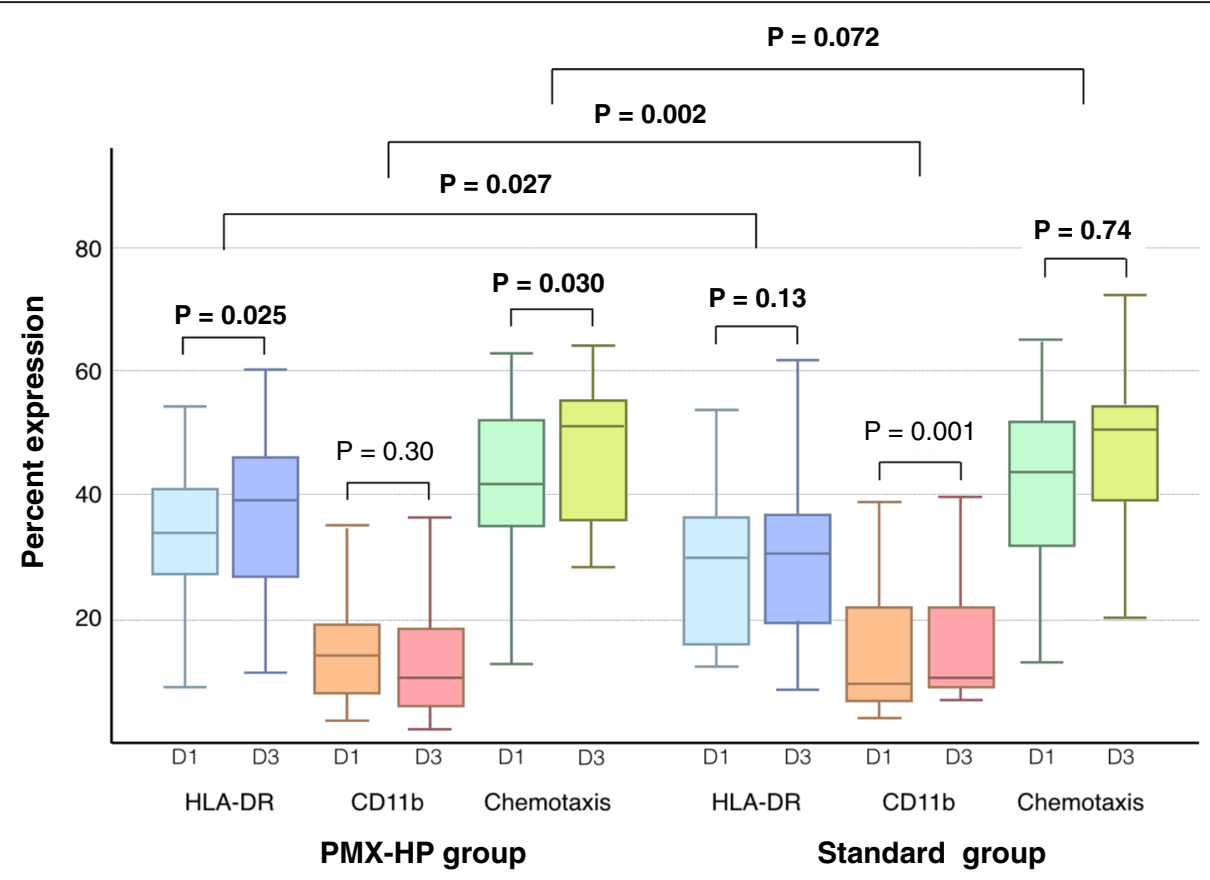

Fig. 2 Comparison of mHLA-DR expression, CD11b expression, and chemotaxis on neutrophil between PMX-HP group and non-PMX-HP group. $\mathrm{D}$ day, mHLA-DR monocytic human leukocyte antigen, PMX-HP polymyxin B hemoperfusion

of neurotoxicity or nephrotoxicity related to polymyxin $B$ were reported.

\section{Discussion}

Key findings

The main finding of our study is that in patients with severe sepsis after PMX-HP treatment, mHLA-DR expression increased and $\mathrm{CD} 11 \mathrm{~b}$ expression decreased compared to standard care. Also, in patients treated with PMX-HP there was a significant increase in neutrophil chemotaxis and a decrease in CVS SOFA score between days 1 and 3 (Table 3 ). Due to our small sample size, we could not calculate the adjusted impact of PMX-HP on 28-day mortality. However, in univariate analysis, we found no significant difference in mortality rates between the PMX-HP and non-PMX-HP groups (Table 4, Additional file 1: Figure S1). We did not encounter any safety issues using PMX-HP. Other secondary outcomes including ICU-free days, mechanical ventilator-free days, RRT, renal recovery, vasopressor-free days, and MAKE 28 were not significantly different between treatment groups on day 28 (Table 3 ).

The improvement in mHLA-DR expression and neutrophil chemotaxis activity (only in the PMX-HP group) after PMX-HP correspond to the "Cytokinetic Theory", one of the new concepts of blood purification, proposed by Peng and by Kellum et al. [27]. This theory suggested that blood purification improved "leukocyte reprogramming" by acting at the cellular level of monocyte and neutrophil function. Data from uncontrolled clinical studies supported the "Cytokinetic Theory" [13, 28]. However, the mechanism of PMX-HP in improving mHLA-DR expression is still unclear. We hypothesized that PMX-HP reduced circulating endotoxin, which is one of the major drivers of intravascular inflammation. This may have led to a reduced intense inflammatory response/cytokine burden in the blood and subsequently to two separate effects-attenuated immunosuppression and restoration of chemokine gradients between the circulation and the site of infection. Although our study could not demonstrate the difference of EAA level on day 3 after treatment (Table 3), there are some possible explanations. First, the production rate of endotoxin might have been higher than the removal rate of endotoxin by PMX-HP. Secondly, the dose of PMX-HP (2 h/ session, 2 days) might not have been enough in some septic patients who had a high endotoxin burden.

Neutrophils play an important role in the inflammatory response in sepsis [5]. Impaired neutrophil function is also associated with subsequent nosocomial infection [29]. The improvement in neutrophil chemotaxis in our study could be explained by the "Cytokinetic Theory". After the direct effect of removing endotoxins and the indirect effect of removing inflammatory mediators from the blood compartment by PMX-HP there might be an increase in the cytokine/chemokine concentration gradient from plasma to infected tissue. Consequently, neutrophils could move toward the site of infection and allow an increase in local 
Table 4 Clinical outcomes by treatment group

\begin{tabular}{|c|c|c|c|}
\hline & PMX-HP group & Non PMX-HP group & $P$ value \\
\hline Overall & $n=29$ & $n=30$ & \\
\hline $\begin{array}{l}\text { Mortality on day } 3 \text {, } \\
n(\%)\end{array}$ & $3(10.3)$ & $10(33.3)$ & 0.058 \\
\hline $\begin{array}{l}\text { Mortality on day } 7 \text {, } \\
n(\%)\end{array}$ & $6(20.7)$ & $12(40.0)$ & 0.11 \\
\hline $\begin{array}{l}\text { Mortality on day } 28, \\
n(\%)\end{array}$ & 17 (58.6) & $15(50.0)$ & 0.51 \\
\hline MAKE $28^{\mathrm{b}}(\%)$ & $22(75.9)$ & $22(73.3)$ & 1.00 \\
\hline Alive on day 28 & $n=13^{c}$ & $n=15$ & \\
\hline ICU-free days (Q1, Q3) & $0(0,10)$ & $0(0,8)$ & 0.99 \\
\hline $\begin{array}{l}\text { Ventilator-free days } \\
(\mathrm{Q} 1, \mathrm{Q} 3)\end{array}$ & $0(0,8)$ & $0(0,0)$ & 0.28 \\
\hline RRT, $n(\%)$ & $7(53.8)$ & $6^{d}(50.0)$ & 0.85 \\
\hline $\begin{array}{l}\text { Serum creatinine } \\
(\mathrm{mg} / \mathrm{dl})(\mathrm{Q} 1, \mathrm{Q} 3)\end{array}$ & $1.4(1.1,2.2)$ & $2.1(1.3,2.5)$ & 0.59 \\
\hline $\begin{array}{l}\text { Vasopressor free } \\
\text { days (Q1, Q3) }\end{array}$ & $0(0,25)$ & $11.5(0,25)$ & 0.47 \\
\hline AKI at baseline & $n=25$ & $n=25$ & \\
\hline Renal recovery ${ }^{\mathrm{e}}, n(\%)$ & $5(20.0)$ & $3(12.0)$ & 0.70 \\
\hline
\end{tabular}

Data represent median (25th percentile, 75th percentile) unless otherwise noted Data measured on day 28 unless otherwise noted

PMX-HP polymyxin B hemoperfusion, ICU intensive care unit, Q1 25th percentile, Q3 75th percentile, $R R T$ renal replacement therapy, $A K I$ acute kidney injury

${ }^{\text {a}}$ Fisher's exact test for categorical variables; Kruskal-Wallis test for continuous variables

${ }^{\mathrm{b}}$ Major adverse kidney events on day 28 , comprised of death, or dialysis dependence, or persistent AKI on day 28

IIn the PMX-HP group one patient died by the end of day 28 , but his values were measured and used in the analysis

${ }^{\mathrm{d}}$ In the non-PMX-HP group, RRT data were available in 12 patients

${ }^{e}$ Renal recovery mean alive, dialysis independence and no AKI on day 28 after enrollment

bacterial clearance [28]. The results from our study indicate that severe sepsis patients, who still suffer from an immunoparalysis state as demonstrated by low mHLA-DR expression or impaired chemotaxis function, might receive benefits from PMX-HP treatment.

$\mathrm{CD} 11 \mathrm{~b}$ is constitutively present on neutrophils. CD11b with CD18 as the CD11b/18 complex is composed of three heterodimers, each having a common subunit noncovalently linked to a different subunit, CD11a, CD11b, and CD11c, and its main activity is the regulation of the adhesive interactions of cells with other cells and with the extracellular matrix. CD11b is rapidly upregulated in response to inflammation and proinflammatory cytokines, and thus considered to represent neutrophil activation [30, 31]. Our study showed that neutrophil activation was stabilized in the PMX-HP group compared to the non-PMX-HP group (Fig. 2, Table 3, $P=0.002$ ).

Two recently published RCTs examining the efficacy of PMX-HP reached different conclusions. The Early Use of Polymyxin B Hemoperfusion in Abdominal Septic Shock (EUPHAS) [32], a single-center RCT in Italy, investigated the role of PMX-HP in severe sepsis/septic shock post abdominal surgery and showed that mortality at 28 days was significantly lower in the PMX-HP group than in the standard treatment group. Conversely, a multicenter trial in France (ABDOMIX study) investigated the role of PMX-HP in the same group of patients and could not find a benefit of PMX-HP on mortality [33]. Of note, there were only 81 of 119 patients (69.8\%) who received two PMX-HP sessions in the ABDOMIX study. Both studies did not use the EAA level to guide PMX-HP initiation. The latest meta-analysis by Fujii et al. [34] included six trials and 857 participants, and could not show the benefit of PMX-HP on 28-day mortality and organ dysfunction scores. Again, most of the included studies did not use the endotoxin level to guide intervention.

\section{Strengths and limitations}

Our study has several strengths. First, this study is the first RCT to explore the role of PMX-HP in improvement of monocyte (defined by mHLA-DR expression) and neutrophil (defined by neutrophil chemotaxis, and CD11b expression) function even though this therapy has been available for more than 25 years. Secondly, we used the EAA to select patients who might receive benefits from PMX-HP. Thirdly, the second PMX-HP session was completed in $90 \%$ of participants, which allowed us to fully test the cellular effect of PMX-HP treatment. Finally, our results support the feasibility and safety of this approach as the basis for a future definitive trial. Currently, there is the largest multicenter randomized trial, Evaluating the Use of Polymyxin B Hemoperfusion in a Randomized controlled trial of Adults Treated for Endotoxemia and Septic shock (EUPHRATES), which finished in 2017 but is as yet unpublished. This study aimed to prove the concept of using a biomarker, EAA, to identify the patients who will receive the most benefit from PMX-HP treatment [35].

There are several limitations to our study. First, due to the nature of the study, this single-center study was an unblinded RCT. However, the robust standard protocol for sepsis treatment and the high compliance rates minimize the risk of bias in sepsis treatment. Secondly, the number of participants was rather small (30 in each group), possibly leading to insufficient power for detecting differences in secondary endpoints. Thirdly, the rate of RRT was higher in the PMX-HP group which could affect physiological endpoints. However, even with the imbalance in RRT rate, mHLA-DR expression increased in the PMX-HP group compared to the non-PMX-HP group. Moreover, we performed further analysis using a general linear model (GLM) to model the outcome variable in log scale adjusting for RRT status, and mHLA-DR on day 3 in PMX-HP patients was still significantly higher than in non-PMX-HP patients $(38.3 \%$ 
vs $30.2 \%, P=0.042$ ) (Table 1 ). Lastly, although EAA was considered the only clinically available technique to detect endotoxin activity, the lack of a quantitative technique for endotoxin levels in humans continues to be problematic. EAA, a ratio of activity, has not been validated versus a quantitative measurement in a large population of septic patients. However, with the current evidence, this EAA seems to be a promising method. EAA has shown a relationship with lipopolysaccharide (LPS) concentration [17, 36]. LPS is well known to cause an inflammatory response and organ dysfunction. Moreover, EAA can reflect the severity of sepsis and correlates with clinical outcome [19, 37, 38]. Lastly, EAA is approved by the US FDA and received a CE marked assay.

\section{Conclusions}

PMX-HP showed the potential to improve immunoparalysis status in severe sepsis/septic shock patients and should be considered as a potential treatment strategy for this specific setting.

\section{Additional file}

Additional file 1: Table S1. Monocyte and neutrophil counts stratified by treatment group. Figure S1. Survival curves for PMX-HP and non-PMXHP groups (blue line, PMX-HP; red line, non-PMX-HP). Kaplan-Meier curve for probability of survival to day 28. PMX-HP polymyxin B hemoperfusion. (DOCX $39 \mathrm{~kb})$

\section{Abbreviations}

CVS: Cardiovascular; EAA: Endotoxin activity assay; FMLP: Formyl methionylphenylalanine; MHC: Major histocompatibility complex; mHLA-DR: Monocytic human leukocyte antigen; ICU: Intensive care unit; LPS: Lipopolysaccharide; PMN: Polymorphonuclear cells; PMX-HP: Polymyxin B hemoperfusion; ROS: Reactive oxygen species; RRT: Renal replacement therapy; SOFA: Sequential Organ Failure Assessment

\section{Acknowledgements}

This study is part of a project supported by the Excellence Center for Critical Care Nephrology, King Chulalongkorn Memorial Hospital. The authors thank the staff, fellows, nurses, and research coordinators at Chulalongkorn University. They also thank Miss Sasipha Tachaboon, MSc, medical technologist Miss Pimnara Peerawaranun for statistical analysis, and Win Kulvichit, MD, for proofreading.

\section{Funding}

The investigator-initiated study was funded by an unrestricted research grant from Ratchadapiseksomphot endowment fund, Faculty of Medicine, Chulalongkorn University.

Toray Industries provided the polymyxin B cartridge and endotoxin activity assay kits for use in this study.

\section{Availability of data and materials}

On reasonable request, data from this study are available from the corresponding author.

\section{Authors' contributions}

NS, ST, NL, CK, SP, NT, KT, KP, SE-O, KT, and JAK were responsible for research design, research conduct, and writing the manuscript. NS was responsible for data analysis. NS, ST, and JAK had primary responsibility for the final content of the manuscript. All authors read and approved the final manuscript.

\section{Ethics approval and consent to participate}

Ethics approval was obtained from the Faculty Ethical Committee (IRB No. 578/56). Written inform consent or agreement to participate was obtained from each patient or the patient's surrogate.

\section{Competing interests}

JAK reports grant support and consulting fees from Baxter and NxStage. Toray Industries provided the polymyxin B cartridge and endotoxin activity assay kits for use in this study.

The company had no influence on the study design or analysis or on the comment of this article. None of the other authors have any disclosures.

\section{Publisher's Note}

Springer Nature remains neutral with regard to jurisdictional claims in published maps and institutional affiliations.

\section{Author details}

${ }^{1}$ Division of Nephrology, Department of Medicine, Faculty of Medicine, Chulalongkorn University, Bangkok 10330, Thailand. ²Excellence Center for Critical Care Nephrology, King Chulalongkorn Memorial Hospital, Thai Red Cross Society, Bangkok, Thailand. '3 Department of Critical Care Medicine, The Center for Critical Care Nephrology, CRISMA, University of Pittsburgh School of Medicine, Pittsburgh, PA, USA

Received: 9 February 2018 Accepted: 21 May 2018

Published online: 26 October 2018

\section{References}

1. Angus DC, Linde-Zwirble WT, Lidicker J, Clermont G, Carcillo J, Pinsky MR. Epidemiology of severe sepsis in the United States: analysis of incidence, outcome, and associated costs of care. Crit Care Med. 2001;29(7):1303-10.

2. Fleischmann C, Scherag A, Adhikari NK, Hartog CS, Tsaganos T, Schlattmann $P$, Angus DC, Reinhart K. Assessment of global incidence and mortality of hospital-treated Sepsis. Current estimates and limitations. Am J Respir Crit Care Med. 2016;193(3):259-72.

3. Vincent JL, Marshall JC, Namendys-Silva SA, François B, Martin-Loeches I, Lipman J, Reinhart K, Antonelli M, Pickkers P, Njimi H, et al. Assessment of the worldwide burden of critical illness: the intensive care over nations (ICON) audit. Lancet Respir Med. 2014;2(5):380-6.

4. Aoki H, Kodama M, Tani T, Hanasawa K. Treatment of sepsis by extracorporeal elimination of endotoxin using polymyxin B-immobilized fiber. Am J Surg. 1994;167(4):412-7.

5. Hanasawa K, Tani T, Kodama M. New approach to endotoxic and septic shock by means of polymyxin B immobilized fiber. Surg Gynecol Obstet. 1989;168(4):323-31.

6. Tsuzuki H, Tani T, Ueyama H, Kodama M. Lipopolysaccharide: neutralization by polymyxin B shuts down the signaling pathway of nuclear factor kappaB in peripheral blood mononuclear cells, even during activation. J Surg Res. 2001;100(1):127-34.

7. Wang Y, Liu Y, Sarker KP, Nakashima M, Serizawa T, Kishida A, et al. Polymyxin $B$ binds to anandamide and inhibits its cytotoxic effect. FEBS Lett. 2000:470(2):151-5.

8. Tani T, Hanasawa K, Kodama M, Imaizumi H, Yonekawa M, Saito M, Ikeda T, Yagi Y, Takayama K, Amano I, et al. Correlation between plasma endotoxin, plasma cytokines, and plasminogen activator inhibitor-1 activities in septic patients. World J Surg. 2001;25(5):660-8

9. Nakamura T, Ebihara I, Shimada N, Shoji H, Koide H. Modulation of plasma metalloproteinase-9 concentrations and peripheral blood monocyte mRNA levels in patients with septic shock: effect of fiber-immobilized polymyxin B treatment. Am J Med Sci. 1998;316(6):355-60.

10. Ono S, Tsujimoto H, Matsumoto A, Ikuta S, Kinoshita M, Mochizuki H. Modulation of human leukocyte antigen-DR on monocytes and CD16 on granulocytes in patients with septic shock using hemoperfusion with polymyxin B-immobilized fiber. Am J Surg. 2004;188(2):150-6.

11. Hotchkiss RS, Monneret G, Payen D. Sepsis-induced immunosuppression: from cellular dysfunctions to immunotherapy. Nat Rev Immunol. 2013; 13(12):862-74.

12. Wakefield CH, Carey PD, Foulds S, Monson JR, Guillou PJ. Changes in major histocompatibility complex class $\|$ expression in monocytes and T cells of patients developing infection after surgery. Br J Surg. 1993;80(2):205-9. 
13. Monneret G, Lepape A, Voirin N, Bohé J, Venet F, Debard AL, Thizy H, Bienvenu J, Gueyffier F, Vanhems P. Persisting low monocyte human leukocyte antigen-DR expression predicts mortality in septic shock. Intensive Care Med. 2006;32(8):1175-83.

14. Medzhitov R, Schneider DS, Soares MP. Disease tolerance as a defense strategy. Science. 2012;335(6071):936-41.

15. Levy MM, Fink MP, Marshall JC, Abraham E, Angus D, Cook D, Cohen J, Opal SM, Vincent JL, Ramsay G. 2001 SCCM/ESICM/ACCP/ATS/SIS International Sepsis Definitions Conference. Crit Care Med. 2003;31(4):1250-6.

16. Rhodes A, Evans LE, Alhazzani W, Levy MM, Antonelli M, Ferrer R, Kumar A Sevransky JE, Sprung CL, Nunnally ME, et al. Surviving Sepsis Campaign: International Guidelines for Management of Sepsis and Septic Shock: 2016 Intensive Care Med. 2017:43(3):304-77.

17. Romaschin AD, Harris DM, Ribeiro MB, Paice J, Foster DM, Walker PM, Marshall JC. A rapid assay of endotoxin in whole blood using autologous neutrophil dependent chemiluminescence. J Immunol Methods. 1998; 212(2):169-85

18. Romaschin AD, Klein DJ, Marshall JC. Bench-to-bedside review: clinical experience with the endotoxin activity assay. Crit Care. 2012;16(6):248.

19. Marshall JC, Foster D, Vincent JL, Cook DJ, Cohen J, Dellinger RP, Abraham E, Brett SJ, Smith T, Mehta S, et al. Diagnostic and prognostic implications of endotoxemia in critical illness: results of the MEDIC study. J Infect Dis. 2004; 190(3):527-34.

20. Tavares-Murta BM, Zaparoli M, Ferreira RB, Silva-Vergara ML, Oliveira CH, Murta EF, Ferreira SH, Cunha FQ, et al. Failure of neutrophil chemotactic function in septic patients. Crit Care Med. 2002;30(5):1056-61.

21. Okamura Y, Yokoi H. Development of a point-of-care assay system for measurement of presepsin (sCD14-ST). Clin Chim Acta. 2011;412:2157-61.

22. Vincent $J$, Moreno R, Takala J, Willatts S, De Mendonca A, Bruining H, Reinhart CK, Suter PM, Thijs LG. The SOFA (Sepsis-related organ failure assessment) score to describe organ dysfunction/failure. On behalf of the working group on Sepsis-related problems of the European Society of Intensive Care Medicine. Intensive Care Med. 1996;22(7):707-10.

23. Shore S, Nelson DP, Pearl JM, Manning PB, Wong H, Shanley TP, Keyser T, Schwartz SM. Usefulness of corticosteroid therapy in decreasing epinephrine requirements in critically ill infants with congenital heart disease. Am J Cardiol. 2001;88(5):591-4.

24. Wernovsky G, Wypij D, Jonas RA, Mayer JE Jr, Hanley FL, Hickey PR, Walsh AZ, Chang AC, Castañeda AR, Newburger JW, et al. Postoperative course and hemodynamic profile after the arterial switch operation in neonates and infants. A comparison of low-flow cardiopulmonary bypass and circulatory arrest. Circulation. 1995;92(8):2226-35.

25. Zuppa AF, Nadkarni V, Davis L, Adamson PC, Helfaer MA, Elliott MR, Abrams J, Durbin D. The effect of a thyroid hormone infusion on vasopressor support in critically ill children with cessation of neurologic function. Crit Care Med. 2004;32(11):2318-22

26. Tsujimoto H, Ono S, Hiraki S, Majima T, Kawarabayashi N, Sugasawa H, Kinoshita M, Hiraide H, Mochizuki H. Hemoperfusion with polymyxin Bimmobilized fibers reduced the number of CD16+ CD14+ monocytes in patients with septic shock. J Endotoxin Res. 2004;10(4):229-37.

27. Rimmele T, Kellum JA. Clinical review: blood purification for sepsis. Crit Care. 2011;15(1):205.

28. Call DR, Nemzek JA, Ebong SJ, Bolgos GL, Newcomb DE, Remick DG. Ratio of local to systemic chemokine concentrations regulates neutrophil recruitment. Am J Pathol. 2001;158(2):715-21.

29. Stephan F, Yang K, Tankovic J, Soussy CJ, Dhonneur G, Duvaldestin P, Brochard L, Brun-Buisson C, Harf A, Delclaux C. Impairment of polymorphonuclear neutrophil functions precedes nosocomial infections in critically ill patients. Crit Care Med. 2002;30(2):315-22.

30. van Spriel AB, Leusen JH, van Egmond M, Dijkman HB, Assmann KJ, Mayadas TN, et al. Mac-1 (CD11b/CD18) is essential for fc receptor-mediated neutrophil cytotoxicity and immunologic synapse formation. Blood. 2001; 97(8):2478-86.

31. Aosasa S, Ono S, Mochizuki H, Tsujimoto H, Osada S, Takayama E, Seki S, Hiraide $\mathrm{H}$. Activation of monocytes and endothelial cells depends on the severity of surgical stress. World J Surg. 2000;24(1):10-6.

32. Cruz DN, Antonelli M, Fumagalli R, Foltran F, Brienza N, Donati A, alcangi V, Petrini F, Volta G, Bobbio Pallavicini FM, et al. Early use of polymyxin B hemoperfusion in abdominal septic shock: the EUPHAS randomized controlled trial. JAMA. 2009:301(23):2445-52.
33. Payen DM, Guilhot J, Launey Y, Lukaszewicz AC, Kaaki M, Veber B, Pottecher J, Joannes-Boyau O, Martin-Lefevre L, Jabaudon M, et al. Early use of polymyxin B hemoperfusion in patients with septic shock due to peritonitis: a multicenter randomized control trial. Intensive Care Med. 2015:41(6):975-84.

34. Fujii T, Ganeko R, Kataoka Y, Furukawa TA, Featherstone R, Doi K, et al. Polymyxin B-immobilized hemoperfusion and mortality in critically ill adult patients with sepsis/septic shock: a systematic review with meta-analysis and trial sequential analysis. Intensive Care Med. 2018;44(2):167-78.

35. Dellinger P. EUPHRATES: evaluating the use of polymyxin B hemoperfusion in a randomized controlled trial of adults treated for endotoxemia and septic shock. Toronto: Canadian Critical Care Forum; 2016.

36. Romaschin AD, Obiezu-Forster CV, Shoji H, Klein DJ. Novel insights into the direct removal of endotoxin by polymyxin B hemoperfusion. Blood Purif. 2017;44(3):193-7.

37. Ikeda T, Ikeda K, Suda S, Ueno T. Usefulness of the endotoxin activity assay as a biomarker to assess the severity of endotoxemia in critically ill patients. Innate Immun. 2014;20(8):881-7.

38. Adamik B, Smiechowicz J, Jakubczyk D, Kübler A. Elevated serum PCT in septic shock with endotoxemia is associated with a higher mortality rate. Medicine (Baltimore). 2015;94(27):e1085.

\section{Ready to submit your research? Choose BMC and benefit from:}

- fast, convenient online submission

- thorough peer review by experienced researchers in your field

- rapid publication on acceptance

- support for research data, including large and complex data types

- gold Open Access which fosters wider collaboration and increased citations

- maximum visibility for your research: over $100 \mathrm{M}$ website views per year

At BMC, research is always in progress.

Learn more biomedcentral.com/submissions 